\title{
Tuberculosis and Nanotechnology: Where shall the Twain meet?
}

Nusrat Shafiq, Ritika Kondel, Samir Malhotra

\begin{abstract}
Tuberculosis remains among the commonest infectious diseases in the developing world and is a major source of morbidity and mortality. Conventional treatments typically consist of long-term therapy with multiple drugs which often leads to poor patient compliance as well as adverse effects. Poor compliance is in turn a major cause for the development of multidrug resistant (MDR) tuberculosis for which there are few treatment options. Therefore, there is an urgent need for improved and more effective therapies. There are very few new drugs for tuberculosis in the pipeline, and a major current area of research is development of novel formulations. Another key aspect is to prevent the spread of the disease by developing rapid diagnostic methods. Here, we describe advances in nanotechnology for improving the pharmacological profile of antitubercular drugs (substantial work in our institution) and microtechnology diagnostics for tuberculosis (TB).
\end{abstract}

Keywords: Tuberculosis, Nanotechnology.

How to cite this article: Shafiq N, Kondel R, Malhotra S. Tuberculosis and Nanotechnology: Where shall the Twain meet? J Postgrad Med Edu Res 2013;47(4):188-192.

Source of support: Nil

Conflict of interest: None declared

\section{INTRODUCTION}

It is estimated that nearly 9 million new cases of tuberculosis are added every year worldwide. ${ }^{1}$ In a recent Indian Council of Medical Research, survey undertaken in our institution, involving 2100 patients, the prevalence of multidrug resistant tuberculosis (MDR-TB) was found to be very high in newly diagnosed cases $(9 \%)$ as compared to WHO data (3-5\%); prevalence was even higher (27\%) in previously treated patients as these patients stop taking drugs in-between. ${ }^{2}$

Research in tuberculosis is a priority area for several funding agencies not only in developing countries but also in developed ones including private funding agencies. A recent systematic review ${ }^{3}$ listed the priority areas in research in tuberculosis identifying 33 research agendas for tuberculosis published between 1998 and 2010. Two clear research priorities emerged from this systematic review: development and testing of both new drugs and treatment regimens and new diagnostic tests for tuberculosis. According to a treatment action group report on tuberculosis research and development funding, investments in tuberculosis research and development in 2007 were mostly in drug development (US\$170 million, 35\%). ${ }^{4}$ While the focus of drug development has been finding new molecules for novel drug targets, development of vaccines, finding strategies to improve the pharmacological profile of existing drugs, the focus of diagnostics has been to develop sensitive, specific and, if possible, point of care diagnostic strategies.

Nanotechnology, a science with myriad applications is increasingly being recognised as a platform technology for improving drug and vaccine delivery as well as for improving diagnostics. The technology itself is variegated and several options are available for instance, nanosuspensions, nanoemulsions, niosomes and polymeric or nonpolymeric nanoparticles. A detailed discussion on these systems is not the focus of this review and thus only the salient features of each are given below.

\section{Nanosuspensions}

Nanosuspensions are colloidal dispersions of nanosized drug particles stabilized by the surfactants. ${ }^{5}$ Their major advantages are enhancement of the solubility and bioavailability of drugs, suitability for hydrophilic drugs, high drug loading, possibility of dose reduction, increased physical and chemical stability of drugs and passive drug targeting.

\section{Nanoemulsions}

Nanoemulsions are oil-in-water (o/w) emulsions of submicron size ranging from 50 to $1000 \mathrm{~nm}$. They serve as vehicles for delivery of active pharmaceutical ingredients as well as other bioactives and are designed to address some of the problems associated with conventional drug delivery systems, such as low bioavailability and noncompliance. ${ }^{6}$

\section{Niosomes}

Niosomes are essentially nonionic surfactant based multilamellar or unilamellar vesicles in which an aqueous solution of solute is entirely enclosed by a membrane resulting from the organization of surfactant macromolecules as bilayer. It represents a promising drug delivery module due to its ability to encapsulate different type of drugs within their multienvironmental structure. These systems have been evaluated as immunological adjuvants, anti-cancer/antiinfective drug targeting agents, carriers of anti-inflammatory drugs and have also been used in diagnostic imaging. ${ }^{7}$ 


\section{Polymeric and Nonpolymeric Nanoparticles}

Biodegradable nanoparticles (NPs) have been extensively exploited over the past few decades for utilization as effective drug delivery devices. Nanoparticles generally vary in size from 10 to $1000 \mathrm{~nm}$. The drug is dissolved, entrapped, encapsulated or attached to a matrix. Many polymeric NPs have been developed for the purpose of sustained release and for targeted drug delivery. ${ }^{8}$ Several nonpolymeric NPs have recently been developed for photodynamic therapy as an emerging alternative to chemotherapy and radiotherapy for cancer treatment. Depending on their chemical composition, these can be used for diagnosis and therapy due to the selective accumulation of the photosensitizer in cancer cells. ${ }^{9}$

For more detailed description as well as to understand better the challenges involved in the development of such nanosystems, readers may refer to an earlier review by us. ${ }^{10}$

\section{USE OF NANOTECHNOLOGY FOR IMPROVING PHARMACOLOGICAL PROFILE OF EXISTING DRUGS}

Compliance to prescribed regimens for tuberculosis is the backbone of tuberculosis control programs world over. The prolonged pharmacotherapy and the pill burden can lower compliance and adherence to administration schedules remain the main reasons for therapeutic failure and contribute to the development of MDR-tuberculosis. Reduction of pill burden can be achieved by employing two strategies - either by increasing the duration of action of a drug or by developing fixed dose combination or a combination of both the strategies.

Polymeric nanoparticles have been extensively evaluated for achieving the sustained release effect. Pioneering experimental work done in our institution has shown that encapsulation of first-line antitubercular drugs rifampicin, isoniazid, pyrazinamide and ethambutol in a polymeric system can be achieved with decent encapsulation efficiency as well as loading; leading to a sustained-release profile so much so that these drugs could be detected in the plasma for up to 9 days after a single administration of such nanoencapsulated drugs. ${ }^{11}$ In contrast, after free drug administration, drugs could be detected for less than 24 hours.

Besides improving the pharmacokinetic profile, preclinical studies in infected mice indicated that five oral doses of drug-loaded nanoparticles administered over 50 days could effectively eliminate the pathogen from different organs similar to or better than 46 daily doses of conventional formulations. ${ }^{12}$ The concept, therefore, is of reducing the dosing frequency with the aim of reducing the pill burden and improving compliance.
In theory, for treatment using the DOTS strategy, such nano-drugs have the potential to drastically reduce the number of visits a patient has to make to the DOTS centre.

\section{Polylactide-coglycolic Acid}

Many researchers including the authors in the abovementioned studies and ourselves have used a polymer named polylactide-coglycolic acid (PLGA). PLGA offers the advantage of being biodegradable and has been approved for human use by US FDA. The other advantages of PLGAbased nanoformulations are their biocompatibility, feasibility of all routes of administration, long shelf-life and versatile degradation kinetics of the polymer. ${ }^{13}$ The polymer acts as a depot and releases the drug slowly over time. Our group is currently working on the scale-up of these nanoformulations in a GMP facility in order to undertake phase I clinical trials of first-line antitubercular drugs in healthy human volunteers.

Keeping in mind the importance of MDR-tuberculosis, our group investigated second line antitubercular drugs on similar lines. In the initial stages, development of nanoformulations of ethionamide and levofloxacin were explored and a similar sustained release action was obtained. The pharmacokinetics of the new formulation of ethionamide and levofloxacin was significantly altered. Ethionamide-loaded nanoparticles provided sustained release of ethionamide for up to 6 days in plasma and 5 to 7 days in tissues while maintaining drug levels above the MIC for 5 days as compared to free ethionamide. ${ }^{14}$ Levofloxacin-loaded nanoparticles also demonstrated a prolonged drug releases profile for up to 4 days in plasma and 6 days in tissues while maintaining drug levels above the MIC for 24 hours in case of encapsulated levofloxacin as compared to 3 hours for free levofloxacin in plasma. ${ }^{15}$

An emerging advantage of PLGA based nanoformulations is their uptake by macrophage and their residence in the same. ${ }^{16}$ Investigators have loaded PLGA based nanoparticles with sufficient rifampicin to efficiently clear macrophages of infection with Mycobacterium bovis BCG. Using fluorescence microscopy and immunoelectron microscopy, in combination with markers for lysosomes, it has been shown that BCG bacteria, as expected, localized to early phagosomes, but at least $90 \%$ of PLGA particles were targeted to and remained in low $\mathrm{pH}$, hydrolase-rich phagolysosomes. Their data collectively argue that PLGA NPs remain membrane-enclosed in macrophages for at least 13 days and degrade slowly. The main implication of this finding was that if the NPs are fabricated with sufficient antibiotic, one dose given after infection is sufficient to efficiently clear the BCG infection after 9 to 12 days treatment. 


\section{Toxicology of Nanoformulations}

The other important concern with antitubercular drugs is their toxicity profile. This concern is more with the second line antitubercular drugs. Two strategies are usually employed to tackle the issue of systemic toxicity-one is that of targeted delivery and another is modifying the release profile of the drug. Both of these strategies have been worked out for pulmonary tuberculosis using nanotechnology. Localized delivery of inhalational alginate-based ${ }^{17}$ and solidlipid nanoformulation ${ }^{18}$ of isoniazid, pyrazinamide and rifampicin have been shown to achieve levels above the minimum inhibitory concentration for 15 days and 7 days respectively without showing any hepatotoxicity when evaluated in animal models.

Our group's work on acute and repeat-dose toxicity studies in animals has shown that these nanoformulations of firstline and secondline antitubercular drugs are not more toxic and, in some cases, even less toxic, than the conventional formulations.

\section{Extrapulmonary Tuberculosis}

After demonstration in landmark work ${ }^{19-21}$ done at our institution, of reduction in recurrence rate with antitubercular therapy in case of serpiginous tuberculosis, it was suggested that like in other conditions of posterior chamber, it is possible to devise a strategy of delivering antitubercular drugs to the posterior chamber using nanotechnology. The fact that a nanotechnology-based implant of dexamethasone which elutes drugs for a period of 3 months has come into clinical use for uveitis, is a good example for translational potential of this strategy. Our group is currently working on developing such a system in collaboration with Punjab University, Chandigarh.

\section{NANOTECHNOLOGY AND VACCINES FOR TUBERCULOSIS}

Although bacillus Calmette-Guerin (BCG) is an effective vaccine for childhood $\mathrm{TB}$, it produces incomplete and variable protection in adult TB. Furthermore, BCG is also an attenuated vaccine that cannot be safely given to the persons infected with human immunodeficiency virus (HIV). Thus, a new and efficient vaccine against TB is urgently required. A few studies have shown that nanoparticlemediated delivery of immunogens may result in improved protection against TB. ${ }^{22,23}$ Protective efficacy of a cationic nanoparticle-based DNA vaccine expressing antigen 85A and early secretory antigen target of Mycobacterium tuberculosis has been demonstrated in murine model. The nanoparticulate system used was $\mathrm{FeO}_{4}$-Glupolyethylenimine (PEI) based and it demonstrated a greater efficacy than the DNA vaccine which was not formulated in the nanosystem.

Specifically, mycobacterial latency antigen, such as Rv1733C, have been used for exploratory evaluation of vaccine. The immunogenicity of a DNA vaccine encoding the MTB latency antigen Rv1733c have been explored for the effect of pulmonary delivery and coformulation with PLGA and PEI on host immunity. The nanoparticles were able to mature human dendritic cells and stimulated them to secrete 11-12 and TNF alpha comparable to levels after lipopolysaccharide stimulation. The vaccine system increased $\mathrm{T}$ cell proliferation and IFN gamma production more potently when applied to lungs directly.

Recently, research has shown the efficacy of a novel nanoparticle-based recombinant DNA vaccine, which contains Esat-6 three T cell epitopes (Esat-6/3e) and fMSlike tyrosine kinase 3 ligand (FL) genes (termed Esat-6/3eFL), and was enveloped with chitosan (CS) nanoparticles (nano-chitosan). ${ }^{24}$ The immunologic and protective efficacy of the nano-chitosan-based DNA vaccine (termed nanoEsat-6/3e-FL) was assessed in mice after intramuscular prime vaccination with the plasmids DNA and nasal boost with the Esat-6/3e peptides. The results showed that the immunized mice remarkably elicited enhanced T-cell responses and protection against Mycobacterium tuberculosis (MTB) H37Rv challenge. These findings indicate that the nanochitosan can significantly elevate the immunologic and protective effects of the DNA vaccine, and the nano-Esat6/3e-FL is a useful vaccine for preventing MTB infection in mice. Chitosan is a Chitosan (CS) is a nontoxic biodegradable and biocompatible polycationic polymer, which can not only bind DNA and protect it from nuclease degradation but also exhibit potential adjuvant properties, such as promoting endocytotic uptake and elevating immune responses. Additionally, chitosan nanoparticles (nanochitosan) possess lower cytotoxicity and more stability.

Nanosized virus-like particles (VLPs) are a particulate vaccine delivery system and can act as adjuvant as well as antigen delivery through enhancing the antigen uptake by antigen presenting cells. ${ }^{25}$ VLPs have the advantage of better stability and capacity to transport across biological barriers. These particles are able to generate CD4, CD8 and TH1 responses. Hepatitis B virus core proteins-VLPs are in the size range of 20 to $200 \mathrm{~nm}$ and are effective in generating both cellular and humoral responses.

\section{NANOTECHNOLOGY AND DIAGNOSIS OF TUBERCULOSIS}

To develop diagnostic tools for infectious diseases at resource-limited settings, the World Health Organization 
(WHO) has established a set of guidelines: (i) affordable, (ii) sensitive, (iii) specific, (iv) user-friendly, (v) rapid and robust, (vi) equipment-free and (vii) delivery to those who need it, leading to the acronym 'ASSURED'. Further, pointof-care (POC) diagnostics offer great potential to detect and monitor infectious diseases at resource-limited settings, because POC diagnostics can be taken to remote locations, decreasing the need for large decentralized diagnostics facilities. Desired characteristics of POC diagnostic technologies include (i) disposability, (ii) cost-effectiveness, (iii) ease of use and (iv) portability.

Nano/microfluidic technologies which are based on the fluid flow in and around nanoscale objects and have a potential for serving the purpose of POC diagnosis. This technology has been used for helping in various aspects of diagnosis, such as sample preparations, blood flow fractionation, nucleic acid extraction and purification of small molecules.

Sheehan et al reported a polymerase chain reaction (PCR)-based Mycobacterium tuberculosis diagnostic device designed as a microfluidic device for DNA amplification. ${ }^{26}$ They described a noncontact thermocycling approach using low power halogen lamp for DNA amplification from the pathogen. In addition, sample and reagent consumption is reduced and the lower thermal mass decreases the required time for PCR from hours to minutes. Lee et al have developed a portable microfluidic nuclear magnetic resonance (NMR) biosensor for rapid, quantitative and multiplexed detection of biological targets, such as bacteria, cancer biomarkers and TB. ${ }^{27}$ In this system, magnetic nanoparticles served as a proximity sensor that bound to target biomolecules and subsequently formed soluble nanoscale clusters which led to NMR signal changes.

A rapid nanogold assay prototypes for specific detection of $M$. tuberculosis complex has recently been reported. ${ }^{28}$ Detection limits were $1 \mathrm{ng}$ for PCR product and $40 \mathrm{ng}$ for genomic DNA. The nano-gold prototype detected 45 positive genomic DNA samples which were also positive with automated liquid culture system (BACTEC ${ }^{\text {TM }}$ MGITTM $^{\mathrm{T}}$ ) and semi-nested PCR (100\% concordance). Following DNA extraction, using standard procedures, the TB nano-gold prototype turnaround time is about 1 hour. The authors concluded that the assay is simple, sensitive and rapid and can be substitute PCR-based detection. Though POC applicability was not emphasized, it could be a good detection system for M. tuberculosis.

\section{CONCLUSION}

The current review demonstrates the translational potential of nanotechnology to address the various challenges associated with the management of tuberculosis and improve current therapeutic strategies. Particle engineering has been shown to lead to improved pharmacokinetic profile in animal models without leading to an increase in toxicity. Enhancement of the immune system for prophylactic use of a novel NP-based DNA vaccine delivery system is an exciting new strategy. Nanoparticles as a diagnostics tool include highly sensitive nanoparticle tests which can potentially address many of the challenges outlined by the World Health Organization for the delivery of rapid and effective point-of-care diagnostics.

\section{REFERENCES}

1. WHO. Global tuberculosis control: a short update to the 2009 report. WHO/HTM/TB/2009426. Geneva: World Health Organization 2009. Available at: http://whqlibdoc.who. int/publications/2009/9789241598866_eng.pdf (accessed July 1, 2010).

2. Sethi S, Mewara A, Dhatwalia SK, Singh H, Yadav R, Singh K, Gupta D, Wanchu A, Sharma M. Prevalence of multidrug resistance in Mycobacterium tuberculosis isolates from HIV seropositive and seronegative patients with pulmonary tuberculosis in north India. BMC Infect Dis 2013 Mar 15;13:137.

3. Rylance J, Pai M, Lienhardt C, Garner P. Priorities for tuberculosis research: a systematic review. Lancet Infect Dis 2010 Dec; 10(12):886-889.

4. Agarwal N. Tuberculosis research and development: a critical analysis of funding trends 2005-2007-an update. New York: Treatment Action Group, 2009 March. Available at: http://www. Treatmentactiongroup.org/uploadedFiles/About/Publications/ TAG_Publications/2009/TAG\%20TB\%202009\%20webversion2.pdf (accessed July 1, 2010).

5. Patel VR, Agrawal YK. Nanosuspension: an approach to enhance solubility of drugs. J Adv Pharm Technol Res 2011 Apr; 2(2):81-87.

6. Lovelyn C, Attama AA. Current state of nanoemulsions in drug delivery. JBNB 2011;2:629-639.

7. Uchegbu IF, Vyas SP. Nonionic surfactant based vesicles (niosomes) in drug delivery. International J Pharmaceutics 1998 Oct;172(1-2):33-70.

8. Soppimath KS, Aminabhavi TM, Kulkarni AR, Rudzinski WE. Biodegradable polymeric nanoparticles as drug delivery devices. J Control Release 2001 Jan 29;70(1-2):1-20.

9. Chouikrat R, Seve A, Vanderesse R, Benachour H, BarberiHeyob M, Richeter S, et al. Nonpolymeric nanoparticles for photodynamic therapy applications: recent developments. Curr Med Chem 2012;19(6):781-792.

10. Kumar G, Shafiq N, Malhotra S. Drug-loaded PLGA nanoparticles for oral administration: fundamental issues and challenges ahead. Crit Rev Ther Drug Carrier Syst 2012;29:149-182.

11. Pandey R, Zahoor A, Sharma S, Khuller GK. Nanoparticle encapsulated antitubercular drugs as a potential oral drug delivery styem against murine tuberculosis. Tuberculosis 2003; 83:373-378.

12. Sharma A, Pandey R, Sharma S, Khuller GK. Chemotherapeutic efficacy of poly (DL-lactide-co-glycolide) nanoparticle encapsulated antitubercular drugs at sub-therapeutic dose against experimental tuberculosis. Int J Antimicrob Agents 2004;24: 599-604. 
13. Geperina S, Kisich K, Iseman MD, Heifets L. The potential advantages of nanoparticles drug delivery systems in chemotherapy of tuberculosis. Am J RespCrit Care Med 2005; 172:1487-1490.

14. Kumar G, Malhotra S, Shafiq N, Pandhi P, Khuller GK, Sharma S. In vitro physicochemical characterization and short term in vivo tolerability study of ethionamide loaded PLGA nanoparticles: potentially effective agent for multidrug resistant tuberculosis. J Microencapsul 2011;28(8):717-728.

15. Kumar G, Sharma S, Shafiq N, Khuller GK, Malhotra S. Optimization, in vitro in vivo evaluation, and short-term tolerability of novel levofloxacin-loaded PLGA nanoparticle formulation. J Pharm Sci 2012 Jun;101(6):2165-2176.

16. Kalluru R, Fenaroli F, Westmoreland D, Ulanova L, Maleki A, Roos N, et al. Polylactide-co-glycolide rifampicin nanoparticles efficiently clear Mycobacterium bovis BCG infection in macrophages and remain membrane-bound in phago-lysosomes. J Cell Sci 2013 July 15;126(pt 14):3043-3054.

17. Zahoor A, Sharma S, Khuller GK. Inhalable alginate nanoparticles as antitubercular drug carriers against experimental tuberculosis. Int J Antimicrob Agents 2005;26:298-303.

18. Pandey R, Khuller GK. Solid lipid particle-based inhalable sustained drug delivery system against experimental tuberculosis. Tuberculosis 2005;85:227-234.

19. Gupta A, Bansal R, Gupta V, Sharma A, Bambery P. Ocular signs predictive of tubercular uveitis. Am J Ophthalmol 2010; 149:562-570.

20. Gupta V, Bansal R, Gupta A. Continuous progression of tubercular serpiginous-like choroiditis after initiating antituberculosis treatment. Am J Ophthalmol 2011;152:857-863.

21. Gupta V, Gupta A, Rao NA. Intraocular tuberculosis - an update. Surv Ophthalmol 2007;52:561-587.

22. Yu F, Wang J, Dou J, et al. Nanoparticle based adjuvant for enhanced protective efficacy of DNA vaccine Ag85A-ESAT-6IL-21 against Mycobacterium tuberculosis infection. Nanomedicine 2012;8:1337-1344.

23. Bivas-Benita M, Lin MY, Bal SM, et al. Pulmonary delivery of DNA encoding Mycobacterium tuberculosis latency antigen RV1733C associated to PLGA-PE1 nanoparticles enhances
T cell response in a DNA prime/protein boost vaccination regimen in mice. Vaccine 2009;27:4010-4017.

24. Feng G, Jiang Q, Xia M, Lu Y, Qiu W, Zhao D, Lu L, Peng G, Wang Y. Enhanced immune response and protective effects of nano-chitosan-based DNA vaccine encoding T cell epitopes of Esat-6 and FL against Mycobacterium tuberculosis infection. PLoS One 2013 Apr 23;8(4):e61135.

25. Dhanasooraj D, Kumar RA, Mundayoor S. Vaccine delivery system for tuberculosis based on nano-sized hepatitis B virus core protein particles. Int J Nanomedicine 2013;8:835-843.

26. Ke C, Berney H, Mathewson A, Sheehan MM. Rapid amplification for the detection of Mycobacterium tuberculosis using a non-contact heating method in a silicon microreactor based thermal cycler. Sensors and Actuators B 2004;102: 308-314.

27. Lee H, Sun E, Ham D, Weissleder R. Chip-NMR biosensor for detection and molecular analysis of cells. Nat Med 2008;14: 869-874.

28. Hussain MM, Samir TM, Azzazy HM. Unmodified gold nanoparticles for direct and rapid detection of Mycobacterium tuberculosis complex. Clin Biochem 2013 May;46(7-8):633-637.

\section{ABOUT THE AUTHORS}

\section{Nusrat Shafiq (Corresponding Author)}

Assistant Professor, Department of Pharmacology, Postgraduate Institute of Medical Education and Research, Chandigarh, India Phone: 9478000822, e-mail: nusrat_shafiq@hotmail.com

\section{Ritika Kondel}

Student, Department of Pharmacology, Postgraduate Institute of Medical Education and Research, Chandigarh, India

\section{Samir Malhotra}

Additional Professor, Department of Pharmacology, Postgraduate Institute of Medical Education and Research, Chandigarh, India 